\title{
Spin-to-orbital angular momentum conversion in semiconductor microcavities
}

\author{
F. Manni, ${ }^{1, *}$ K. G. Lagoudakis, ${ }^{1}$ T. K. Paraïso, ${ }^{1}$ R. Cerna, ${ }^{1}$ Y. Léger, ${ }^{1}$ T. C. H. Liew, ${ }^{2}$ I. A. Shelykh, ${ }^{3}$ A. V. Kavokin,${ }^{4,5}$ \\ F. Morier-Genoud, ${ }^{1}$ and B. Deveaud-Plédran ${ }^{1}$ \\ ${ }^{1}$ Institute of Condensed Matter Physics, École Polytechnique Fédérale de Lausanne (EPFL), CH-1015, Lausanne, Switzerland \\ ${ }^{2}$ Institute of Theoretical Physics, École Polytechnique Fédérale de Lausanne (EPFL), CH-1015 Lausanne, Switzerland \\ ${ }^{3}$ Science Institute, University of Iceland, Dunhagi-3, IS-107, Reykjavik, Iceland \\ ${ }^{4}$ Laboratoire Charles Coulomb UMR 5221 CNRS-UM2, Université Montpellier 2, FR-34095 Montpellier Cedex 5, France \\ ${ }^{5}$ Physics and Astronomy School, University of Southampton, Highfield, Southampton, SO171BJ, United Kingdom
}

(Received 1 June 2011; published 24 June 2011)

\begin{abstract}
We experimentally demonstrate a technique for the generation of optical beams carrying orbital angular momentum using a planar semiconductor microcavity. Despite being isotropic systems with no structural gyrotropy, semiconductor microcavities, because of the transverse-electric-transverse-magnetic polarization splitting that they feature, allow for the conversion of the circular polarization of an incoming laser beam into the orbital angular momentum of the transmitted light field. The process implies the formation of topological entities, a pair of optical vortices, in the intracavity field.
\end{abstract}

DOI: 10.1103/PhysRevB.83.241307

PACS number(s): 78.67.-n, 42.25.-p, 42.50.Tx, 42.79.-e

It is well known that photons can carry both an intrinsic spin and orbital angular momentum. ${ }^{1}$ Both the intrinsic spin ${ }^{2}$ and the orbital angular momentum ${ }^{3}$ can generate a torque on macroscopic objects, which could provide the optical drive of micromachines ${ }^{4}$ and Doppler shifts of spinning bodies. ${ }^{5,6}$ It has also been shown that orbital angular momentum can be coherently transferred to atoms,$^{7}$ allowing, in principle, the storage of high-dimensional quantum information. In fact, while the spin angular momentum is restricted to $\pm \hbar$ for each photon, the orbital angular momentum can take any multiple of $\hbar$. Moreover, again in a view of quantum computation applications, entanglement between orbital angular momentum states has been experimentally demonstrated. ${ }^{8}$ Further applications of beams carrying orbital angular momentum appear in microscopy $^{1}$ and ultrasensitive interferometry suitable for gravitational wave detection. ${ }^{9}$

The first-ever implementation of a laser beam carrying orbital angular momentum was achieved by shining a standard laser beam through a system of lenses. ${ }^{10}$ Later developments showed that individual optical components such as spiral wave plates $^{11,12}$ or holograms with forklike dislocations ${ }^{13}$ could achieve the same task. More recently, more compact components based on birefringent materials have been used. ${ }^{14,15}$ It is notable that all known techniques for the creation of beams with orbital angular momentum require an optically inhomogeneous and/or anisotropic material. Strong focusing can also be used to generate such beams, ${ }^{16}$ as accurately modeled by theory. ${ }^{17,18}$

In this Rapid Communication, we demonstrate that conversion of spin into orbital angular momentum can also be achieved, in the linear regime, with a planar semiconductor microcavity ${ }^{19}$ - a compact, layered nanostructure, acting as a single optical element. At first sight, such conversion is unexpected since a semiconductor microcavity is a planar isotropic system, which features no structural gyrotropy, as it is characterized by a diagonal susceptibility tensor in the linear polarization basis. However, microcavities exhibit a polarization splitting between transverse-electric (TE) and transverse-magnetic (TM) polarized modes, ${ }^{20}$ which can be represented by an effective magnetic field, the direction of which depends on the reciprocal space position excited ${ }^{21}$ despite the isotropic nature of the microcavity [Fig. 1(a)]. This directional dependence arises from the choice of a fixed coordinate system when defining the TE-TM basis, and it does not represent a breaking of the rotational symmetry of the system. This effective magnetic field is responsible for polarized pattern formation, ${ }^{22}$ the "all-optical" spin Hall effect, ${ }^{23}$ and has been predicted to allow conversion between spin and orbital angular momentum. ${ }^{24}$ The effect of the TE-TM splitting on a circularly polarized distribution can be understood intuitively from Fig. 1, which shows the Stokes vectors for light excited on a ring in reciprocal space [the Stokes vectors represent the polarization state of a light mode on the Poincaré sphere as shown in Fig. 1(b)]. While a fully circularly polarized distribution would be characterized by Stokes vectors pointing in the vertical direction (white arrows in Fig. 1), their precession about the effective magnetic fields causes them to evolve in different directions. A polarization component of opposite circularity develops and the phase profile can become dependent on the angle, as we will show. The process conserves the total angular momentum (spin + orbital angular momentum) via spin-to-orbital angular momentum (SOAM) conversion. We provide a theoretical model that is able to quantitatively reproduce the experimental observations.

Formally, under a resonant optical excitation, the dynamics of the intracavity field is given by the two-component Schrödinger equation

$$
\begin{aligned}
i \hbar \frac{\partial \psi_{ \pm}(\mathbf{k}, t)}{\partial t}= & E(k) \psi_{ \pm}(\mathbf{k}, t)+\left(\frac{\Delta(k)}{2} e^{\mp 2 i \phi}+\frac{\xi}{2}\right) \psi_{\mp}(\mathbf{k}, t) \\
& +f_{ \pm}(\mathbf{k}, t)
\end{aligned}
$$

where \pm represents the two circularly polarized components of the field $\psi_{ \pm}(\mathbf{k}, t)$. The two-dimensional (2D) in-plane wave vector $\mathbf{k}=(k, \phi)$ is divided into a radial component $k$ and angular component $\phi . E(k)=E_{c}-i \hbar \Gamma$ represents the complex in-plane dispersion of the cavity mode, where the 
(a)
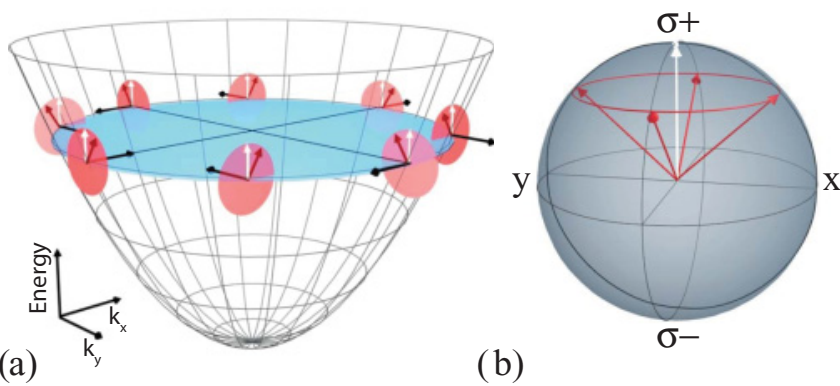

(b)

FIG. 1. (Color online) (a) Illustration of the $k$-dependent effect of the effective magnetic field (TE-TM splitting) on the Stokes vector of the photons [in black: effective magnetic field; in white: initial Stokes vector orientation; in red (dark gray): rotated Stokes vector]. The parabolic dispersion of the cavity mode is sketched in black solid lines. (b) Mapping of the Stokes vectors to the Poincaré sphere [same color code as in (a)].

imaginary component accounts for the decay of photons with rate $2 \Gamma . \Delta(k)$ and $\xi$ represent the strength of the $k$-dependent TE-TM polarization splitting ${ }^{20}$ and a possible additional splitting due to anisotropy, ${ }^{25}$ respectively. The anisotropy term is needed to take into account the small residual linear polarization splitting of real samples. The angular dependence of the TE-TM splitting is equivalent to that represented by the effective magnetic field in Fig. 1(b) of Ref. 21. The pumping term can be written as ${ }^{25}$

$$
\begin{aligned}
& f_{ \pm}(\mathbf{r}, t)=A_{ \pm} e^{-r^{2} / L^{2}} e^{-i \beta r^{2}} e^{-i E_{p} t / \hbar}, \\
& f_{ \pm}(\mathbf{k}, t)=\int f_{ \pm}(\mathbf{r}, t) \frac{i \Gamma e^{-i E_{p} t / \hbar}}{E(k)-E_{p}} d \mathbf{r} e^{i \mathbf{k} \cdot \mathbf{r}},
\end{aligned}
$$

which corresponds to a monochromatic focused Gaussian beam with amplitudes $A_{ \pm}$, spot size $L$, and energy $E_{p}\left(e^{-i \beta r^{2}}\right.$ accounts for the wavefront curvature). Considering a circularly polarized pump with $A_{-}=0$, the steady-state solution of Eq. (1) is given by the equations

$$
\begin{gathered}
\psi_{-}(\mathbf{k}, t)=-\frac{\Delta(k) e^{2 i \phi}+\xi}{E(k)} \psi_{+}(\mathbf{k}, t), \\
\psi_{+}(\mathbf{k}, t)=-\frac{f_{+}(k, t)}{E(k)-\frac{\left[\Delta(k) e^{-2 i \phi}+\xi\right]\left[\Delta(k) e^{2 i \phi}+\xi\right]}{E(k)}} .
\end{gathered}
$$

In the limit $\xi \mapsto 0$, where the anisotropy term is neglected, Eq. (4) becomes independent of $\phi$, that is, the $\sigma_{+}$polarized cavity field (the same polarization as the pump) does not carry orbital angular momentum. However, it is seen from Eq. (3) that the $\sigma_{-}$component carries orbital angular momentum due to the phase profile $e^{2 i \phi}$. This phase winding structure corresponds to vortical entities in the optical transmitted signal that are associated with the generation of an orbital angular momentum. The presence of anisotropy, although not responsible for the SOAM conversion process, can give rise to more complex and exotic structures in the phase profile, as it will be shown below.

To observe the SOAM conversion effect, we perform transmission measurements using the cavity mode of an InGaAs/GaAs microcavity, the same sample used in Ref. 26. Note that although an excitonic resonance exists in the sample, it has negligible effect on the cavity mode resonance since we operate in the regime of very large positive detuning $(\approx 6 \mathrm{meV}$ between the excitonic resonance and the cavity mode resonance at $k=0$ ). We excite in one circular polarization and we detect both circular components of the transmitted signal. In order to demonstrate the existence of vortex excitations, we implement a homodyne detection scheme in the setup, which allows us to retrieve the phase of the signal. ${ }^{27}$ The sample is kept in a liquid helium flow cryostat at low temperature $(\approx 4 \mathrm{~K})$ to ensure the transparency of the GaAs substrate and spacer layer. Note that this constraint only depends on the sample used, and the effect we observe should be reproducible even at room temperature. The laser source used here is a tunable single-mode continuous wave Ti:sapphire laser. The laser is first split into two beams: one is used as the phase reference for the homodyne detection, while the other is prepared in the circular polarization state $\sigma_{+}$and is used to excite the system. This Gaussian-shaped $\sigma_{+}$beam is tightly focused onto the sample using a 0.5 -NA microscope objective. The tight spatial focusing results in a very broad, single-energy excitation in momentum space. Consequently, increasing the excitation energy with respect to the bottom of the cavity mode, we are able to excite a ring with $|\mathbf{k}| \neq 0$ in reciprocal space. This is a key feature that allows us to access a regime where the $k$-dependent TE-TM splitting becomes significant. Moreover, the ring-shaped filtering in $k$ space, intrinsically provided by the system, is such to generate a Bessel beam intensity profile in the transmitted optical field. The real-space transmitted field coming from the sample is collected with another 0.5-NA objective. The two polarizations of opposite circularity $\left(\sigma_{+}, \sigma_{-}\right)$are eventually separated and imaged on a CCD camera using a Wollaston prism. On the same camera, we superimpose the homodyne reference beam in order to obtain interference patterns, from which we extract the phase profiles.

For an excitation energy $2.5 \mathrm{meV}$ above the bottom of the cavity mode, the ring diameter in reciprocal space has a radius of $|\mathbf{k}| \approx 2 \mu m^{-1}$ for which the TE-TM splitting is estimated to be $\Delta(k) \approx 20 \mu \mathrm{eV}$. The polarization-resolved transmitted optical field is shown in Fig. 2(a) for $\sigma_{+}$and in Fig. 2(d) for $\sigma_{-}$polarizations. The transmitted $\sigma_{+}$beam is characterized by a central high-intensity spot surrounded by a series of rings. The opposite circularly polarized $\sigma_{-}$signal shows a much wider central spot, featuring two distinct local minima, located at $(x, y) \approx(0.5,-1) \mu \mathrm{m}$ and $(x, y) \approx(0.5,1) \mu \mathrm{m}$. These minima are attributed to optical vortices because of the SOAM conversion process.

By interfering the transmitted signal with the reference beam, two interferograms are obtained: one for the $\sigma_{+}$and one for the $\sigma_{-}$transmitted beams, shown in Figs. 2(b) and $2(\mathrm{e})$, respectively. Within the central spot of the $\sigma_{-}$signal, we unambiguously identify the presence of two forklike dislocations, highlighted by the white circles in Fig. 2(e). These dislocations coincide with the local density minima of Fig. 2(d), proving the existence of quantized vortices. Note that both interferograms [Figs. 2(b) and 2(e)] feature other forklike dislocations, located at the outer edge of the central spot. These dislocations result from the $\pi$ phase mismatch [see Fig. 2(c)] between the central region and the first density ring and, in fact, do not correspond to optical vortices. 

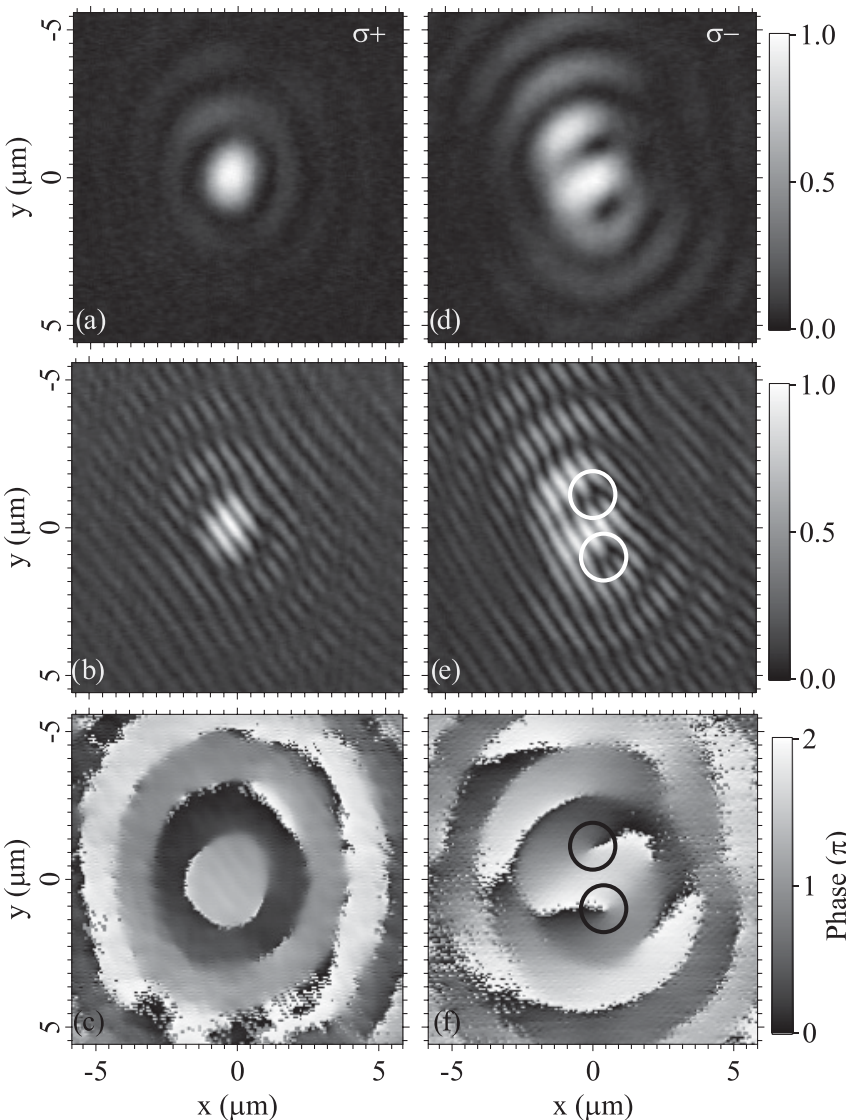

FIG. 2. Real-space profile (a) of the transmitted optical field, interferogram (b), and corresponding phase (c) for the $\sigma_{+}$polarization. Same for the $\sigma_{-}$polarization in (d), (e), and (f), respectively. The white (black) circles mark the position of the pair of optical vortices.

Using digital holography methods ${ }^{28}$ we extract the phase of the transmitted field for both $\sigma_{+}$and $\sigma_{-}$polarizations as shown in Figs. 2(c) and 2(f), respectively. The analysis of the phase confirms that no topological charge is present in the $\sigma_{+}$ transmitted signal [Fig. 2(c)]. Nevertheless, we observe the aforementioned phase structure associated to the ring density profile of Fig. 2(a): each ring is in antiphase with the one before and after. This $\pi$ phase shift is responsible for the forklike dislocations that can be found at the locations $(x, y) \approx$ $(-1.5,1) \mu \mathrm{m}$ and $(x, y) \approx(1,-1) \mu \mathrm{m}$ in the interferogram of Fig. 2(b), which do not correspond to vortices (as they do not coincide with any local density minima).

In the $\sigma_{-}$transmitted signal, the vortex singularities exhibit a linear phase increase as a function of the azimuthal angle, thus confirming that we are in the presence of a pair of optical vortices. The total phase jump along the $\sigma_{-}$outer transmission ring is found to be $4 \pi$, as in the theoretical prediction [see Eq. (3)]. The two vortices present in the $\sigma_{-}$transmission are the signature of the $L=+2$ orbital angular momentum obtained by the conversion of the spin, from $\sigma_{+}(S=+1)$ to $\sigma_{-}(S=$ $-1)$ due to the TE-TM polarization splitting.

Theory predicts that the optical vortices resulting from SOAM conversion are expected to spatially overlap in the absence of anisotropy. When anisotropy is present, the pair of vortices is spatially separated by a distance directly related to the degree of anisotropy in the sample. In general, though, the SOAM conversion is robust and generic on the sample surface. The anisotropy being strongly position dependent allowed us to find positions where the two vortices were closer or farther apart from each other. Moreover, the orientation of the vortex pair is set by the direction of the higher-energy birefringence axis of the sample. The higher-energy polarization caused by birefringence is at $70^{\circ}$ clockwise with respect to the $x$ axis of Fig. 2. Therefore, for polaritons traveling in the $70^{\circ}$ direction (or opposite $250^{\circ}$ direction), the anisotropy has the effect of splitting further TM and TE polarized states. This enhancement of the TE-TM splitting causes more light to couple from the $\sigma_{+}$to $\sigma_{-}$polarization in the $70^{\circ}$ or $250^{\circ}$ direction. The central vortex pair appears as a minimum along the direction of maximum intensity [see Fig. 2(d)], that is, they appear along the birefringence axis.

Finally, to further verify that the observed singularities are a product of the SOAM conversion, we performed the same experiment exciting the parabolic dispersion resonant to $k=$ 0 where the TE-TM splitting is $\Delta(0)=0 \mu \mathrm{eV}$. We did not encounter any phase singularities neither in the $\sigma_{+}$nor the $\sigma_{-}$transmitted signals, thus excluding the role of focusing in the SOAM conversion in our experiment ${ }^{17,18}$ and verifying the dependence of the effect on the TE-TM polarization splitting.

We are able to quantitatively reproduce the experimental findings with the theoretical model introduced above..$^{29}$ The results of the simulations are shown in Fig. 3. The intensity of the optical field is plotted together with the corresponding calculated phase for the $\sigma_{+}$in Figs. 3(a) and 3(b) and for the $\sigma_{-}$polarization in Figs. 3(c) and 3(d), respectively. As it can
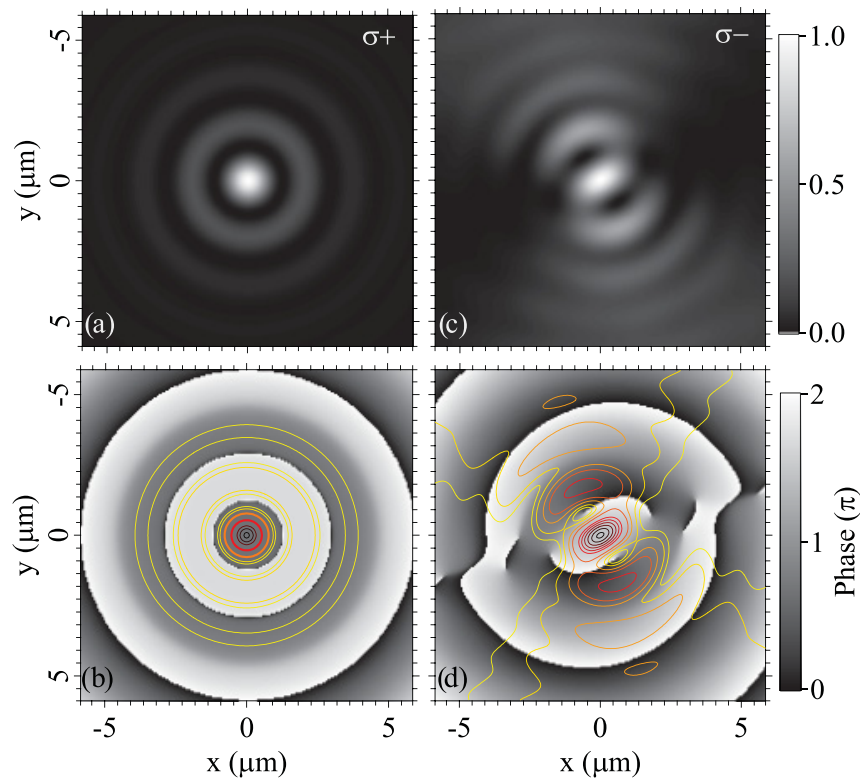

FIG. 3. (Color online) Real-space intensity profile of the intracavity optical field and corresponding phase: (a), (b) for the $\sigma_{+}$and (c), (d) for the $\sigma_{-}$polarizations, respectively. Note: The density contour lines of (a) and (c) are superposed to the corresponding phase in (b) and (d) in order to underline that only two local density minima are present in the $\sigma_{-}$signal, and that they match with two phase windings of $2 \pi$. The presence of anisotropy is responsible for the complex and exotic phase structure. 
be seen in those figures, the theory captures all the features of the experimental findings. In the model, the spatial separation between the two vortices is caused by the $\xi$ anisotropy term.

In this paper, we investigated the creation of beams carrying orbital angular momentum, exploiting an optical microcavity - a 2D optical isotropic and homogeneous element. Our system represents a single compact optical device capable of generation of beams with orbital angular momentum for applications in the control of nanomachines or quantum information. Moreover, being a homogeneous and isotropic optical system, the creation of arrays of elements to generate OAM carrying beams is greatly simplified, not requiring any special nanostructuring. We have demonstrated the creation of a pair of optical vortices, forcing the $\sigma_{-}$ beam to carry a total angular momentum of +2 . This observation proves SOAM conversion occurring between the two orthogonal circular polarizations, which are coupled by the TE-TM splitting featured by the microcavity sample. With the theoretical model presented, we are able to fit the experimental parameters and quantitatively match the results.

This work was supported by the Swiss National Science Foundation through NCCR "Quantum Photonics." *francesco.manni@epfl.ch

${ }^{1}$ S. Franke-Arnold, L. Allen, and M. Padgett, Laser Photonics Rev. 2, 299 (2008).

${ }^{2}$ R. A. Beth, Phys. Rev. 50, 115 (1936).

${ }^{3}$ H. He, M. E. J. Friese, N. R. Heckenberg, and H. RubinszteinDunlop, Phys. Rev. Lett. 75, 826 (1995).

${ }^{4}$ D. G. Grier, Nature (London) 424, 810 (2003).

${ }^{5}$ B. A. Garetz and S. Arnold, Opt. Commun. 31, 1 (1997).

${ }^{6}$ J. Courtial, K. Dholakia, L. Allen, and M. J. Padgett, Phys. Rev. A 56, 4193 (1997).

${ }^{7}$ M. F. Andersen, C. Ryu, P. Cladé, V. Natarajan, A. Vaziri, K. Helmerson, and W. D. Phillips, Phys. Rev. Lett. 97, 170406 (2006).

${ }^{8}$ A. Mair, A. Vaziri, G. Weihs, and A. Zeilinger, Nature (London) 412, 313 (2001).

${ }^{9}$ M. Granata, C. Buy, R. Ward, and M. Barsuglia, Phys. Rev. Lett. 105, 231102 (2010).

${ }^{10}$ L. Allen, M. W. Beijersbergen, R. J. C. Spreeuw, and J. P. Woerdman, Phys. Rev. A 45, 8185 (1992).

${ }^{11}$ S. S. R. Oemrawsingh, E. R. Eliel, J. P. Woerdman, E. J. K. Verstegen, J. G. Kloosterboer, and G. W. t'Hooft, J. Opt. A: Pure Appl. Opt. 6, S288 (2004).

${ }^{12}$ M. W. Beijersbergen, R. P. C. Coerwinkel, M. Kristensen, and J. P. Woerdman, Opt. Commun. 112, 321 (1994).

${ }^{13}$ V. Yu. Bazhenov, M. V. Vasnetsov, and M. S. Soskin, Pis'ma Zh. Eksp. Teor. Fiz. 52, 1037 (1990) [JETP Lett. 52, 429 (1990)].

${ }^{14}$ L. Marrucci, C. Manzo, and D. Paparo, Phys. Rev. Lett. 96, 163905 (2006).

${ }^{15}$ E. Brasselet, N. Murazawa, H. Misawa, and S. Juodkazis, Phys. Rev. Lett. 103, 103903 (2009).

${ }^{16}$ Y. Zhao, J. S. Edgar, G. D. M. Jeffries, D. McGloin, and D. T. Chiu, Phys. Rev. Lett. 99, 073901 (2007).
${ }^{17}$ K. Y. Bliokh, M. A. Alonso, E. A. Ostrovskaya, and A. Aiello, Phys. Rev. A 82, 063825 (2010).

${ }^{18}$ A. Ya. Bekshaev, Cent. Eur. J. Phys. 8, 947 (2010).

${ }^{19}$ A. V. Kavokin, J. J. Baumberg, G. Malpuech, and F. P. Laussy, Microcavities (Oxford University, New York, 2007).

${ }^{20}$ G. Panzarini, L. C. Andreani, A. Armitage, D. Baxter, M. S. Skolnick, V. N. Astratov, J. S. Roberts, A. V. Kavokin, M. R. Vladimirova, and M. A. Kaliteevski, Phys. Rev. B 59, 5082 (1999).

${ }^{21}$ A. V. Kavokin, G. Malpuech, and M. M. Glazov, Phys. Rev. Lett. 95, 136601 (2005).

${ }^{22}$ W. Langbein, I. Shelykh, D. Solnyshkov, G. Malpuech, Yu. Rubo, and A. Kavokin, Phys. Rev. B 75, 075323 (2007).

${ }^{23}$ M. Maragkou, C. Richards, T. Ostatnicky, A. J. D. Grundy, J. Zajak, W. Langbein, and P. Lagoudakis, Opt. Lett. 36, 1095 (2011).

${ }^{24}$ T. C. H. Liew, A. V. Kavokin, and I. A. Shelykh, Phys. Rev. B 75, 241301 (2007)

${ }^{25}$ A. Amo, T. C. H. Liew, C. Adrados, E. Giacobino, A. V. Kavokin, and A. Bramati, Phys. Rev. B 80, 165325 (2009).

${ }^{26}$ T. K. Paraïso, D. Sarchi, G. Nardin, R. Cerna, Y. Leger, B. Pietka, M. Richard, O. El Daïf, F. Morier-Genoud, V. Savona, and B. DeveaudPlédran, Phys. Rev. B 79, 045319 (2009).

${ }^{27}$ G. Nardin, Y. Leger, B. Pietka, F. Morier-Genoud, and B. DeveaudPlédran, Phys. Rev. B 82, 045304 (2010).

${ }^{28}$ K. G. Lagoudakis, T. Ostatnicky, A. V. Kavokin, Yu. Rubo, R. André, and B. Deveaud-Plédran, Science 326, 974 (2009).

${ }^{29}$ Parameters for the simulations: $E_{u p}(k)$ was taken as a parabolic dispersion with effective mass $5 \times 10^{-5}$ of the free electron mass. The pump energy was chosen as $E_{p}=E_{u p}(0)+3 \mathrm{meV}$, which excites a ring in $k$ space at approximately $2 \mu \mathrm{m}^{-1}$ radius. $\Delta(k)$ was taken as parabolic with $\Delta\left(2 \mu \mathrm{m}^{-1}\right)=0.02 \mathrm{meV}$. The other parameters were $\xi=0.02 \mathrm{meV}, L=5.1 \mu \mathrm{m}, \Gamma=0.33 \mathrm{meV}$, and $\beta=0.14 \mu \mathrm{m}^{-2}$. 